\title{
Pressure-Impulse Diagrams for an Elastic- Plastic Member under Confined Blasts
}

\author{
Jonathon Dragos, Chengqing Wu* and Kate Vugts \\ School of Civil and Environmental Engineering, The University of \\ Adelaide, SA, Australia
}

Received on 22 Feb 2013, Accepted on 11 April 2013

\begin{abstract}
Pressure-impulse (P-I) diagrams based on the equivalent single-degreeof-freedom approach (SDOF) have been used during building design in order to assess the effects of blasts on structures. They provide an easy way to describe the likely outcome of the combination of blast pressures and impulses on a particular structural element in a building at the moment an explosion occurs. However, only P-I diagrams of structural members under external blasts have been addressed in current guidelines and previous studies. Due to the complexity of confined scenarios, confined blast pressure-time histories cannot be approximated by simplified representations of pressure-time histories used for external blasts, such as triangular shapes representing linear decay or curves indicating exponential decay. Rather, they should be simplified as bilinear pressure-time histories. Thus, SDOF models which incorporate bilinear blast loads were developed to predict the response of a member with a bilinear, elastic-plastic-hardening, resistance-deflection function. Then using the developed SDOF model, normalised P-I Diagrams for structural members with bilinear resistancedeflection functions under bilinear blasts were generated. These results were then used to undertake a parametric study to investigate the influence of varying blast load shapes and varying bilinear resistancedeflection function shapes on the normalised P-I curves. Also, comparisons against other techniques employed to eliminate pulse load shape effects were also undertaken for bilinear pulse loads and bilinear resistance-deflection function shapes.
\end{abstract}

Key words: pressure-impulse diagrams, SDOF, elastic-plastic members, confined blasts

\section{INTRODUCTION}

Confined environments may occur in many scenarios such as tunnels and subway stations or within car parks and basements of buildings or due to an accidental explosion within an ammunition storage bunker, or in a building with strong glass panels or even in city streets [1-4]. As confined spaces magnify the effects of explosions, the evaluation of confined

*Corresponding author. E-mail address: cheng.wu@adelaide.edu.au 
explosion hazards and their consequence on infrastructure at high risk is important to the safety of people and installations [5-6]. Therefore analysis and design of infrastructure under critical scenarios of confined blast-loading will help protect civil infrastructure. Should a gas line rupture and explode, or a bomb be detonated in a subway, it is important to know that the damage will be contained by the immediately affected reinforced concrete (RC) members and not spread to nearby critical infrastructure, including telecommunications cables, vaults or secure storage spaces. Because the problem of an explosion in confined environments is much more complex than in the free field, confined blast effects on structures are less addressed by any researchers and in the current guidelines such as UFC-3-340-02 [7] and the soon-to-be published ASCE Standard for the Blast Protection of Buildings [8].

Guidelines in UFC-3-340-02 assume two superimposed imaginary peak triangular overpressure pulses, such as in Figure 1, to be a typical overpressure history in confined environments. The first imaginary peak represents the shock pressures and the second imaginary peak represents the gas pressures. As seen in Figure 2, the actual overpressure relationship is highly irregular due to multiple reflections. This pressure-time history was caused by a $1 \mathrm{~kg}$ cylindrical charge placed in the centre of a fully confined cube of volume $4 \mathrm{~m}^{3}$. The cylindrical charge is oriented such that the vertical axis intercepts the pressure gauge. The most recent studies, such as Dragos et al. [9], indicate that it is appropriate to simplify a confined blast pressure-time history to a bilinear pulse shape as shown in Figure 1. However,

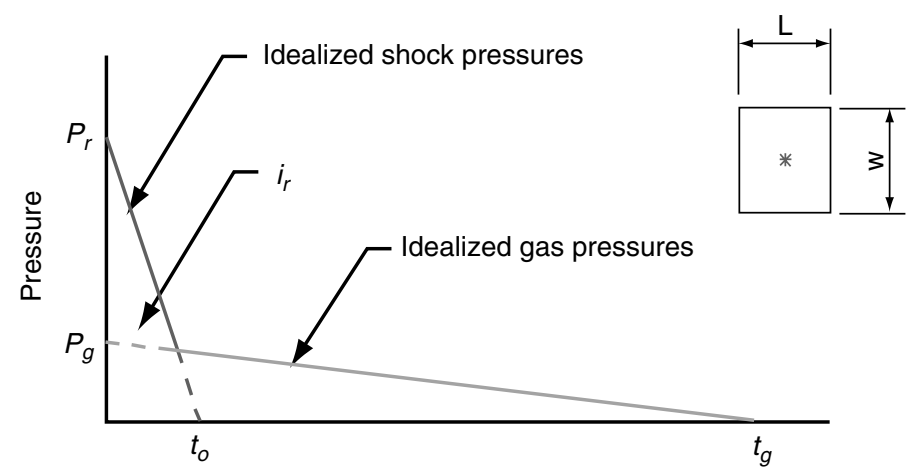

Figure 1. UFC-3-340-02 idealised internal blast model

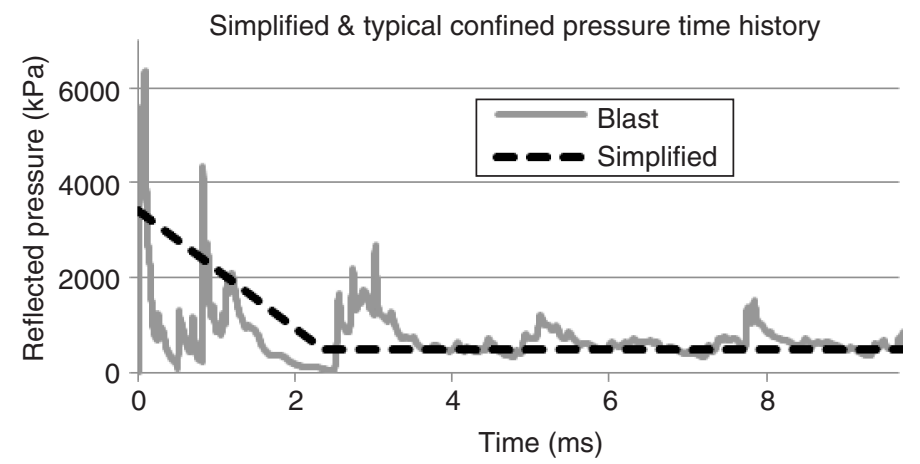

Figure 2. Realistic and simplified record of pressures for internal blasts 
how a confined blast affects the structural response is not addressed in the current guidelines, nor has this been the subject of any previous studies. Therefore, the consequences of confined blasts on high risk infrastructure need to be taken into account in analysis and design of structures.

A pressure-impulse (P-I) diagram is a well-used design tool used by building designers to assess the response of structural members to a specified load. The diagram indicates the combinations of peak reflected pressure and impulse, as from a blast, that will cause the failure or a specific level of damage of a structure or structural member [10]. Krauthammer et al. [11], for example, used a single-degree-of-freedom (SDOF) model to undertake a dimensional analysis of P-I curves for elastic structural members subjected to different pulse loads. This was done to investigate the effect of three pulse shapes (exponential, triangular and rectangular) that result from different sorts of explosives and their placement. The study provided non-dimensional, or normalised, P-I curves for these three pulse load shapes. Li \& Meng [12] also undertook a dimensional analysis of P-I curves for elastic members subjected to the blasts with the aforementioned pulse shapes. They also attempted to eliminate the effects of pulse shape by using an empirical function to describe any elastic non-dimensional or normalised P-I curve. This function contains two coefficients which are both a function of the properties of the associated pulse shape. By curve fitting, Li \& Meng [12] attempted to develop empirical equations for these coefficients based on the properties of the pulse shape. Li \& Meng [13] extended the dimensional analysis studies, focussing on exponential, triangular, and rectangular shaped pulses acting on elastic-perfectly-plastic structural members. Fallah \& Louca [14] attempted to extend the studies even further, again focussing on exponential, triangular, and rectangular shaped pulse loads acting on bilinear elastic plastic hardening/softening structural members. Youngdahl [15] introduced parameters which can be used to eliminate the pulse shape effects of normalised P-I curves for rigid-plastic structural members. According to Fallah \& Louca [14], Youngdahl parameters can also be used to eliminate pulse shape effects for rigid-plastic-hardening structural members. However, they also stated that these parameters cannot be used to eliminate pulse shape effects of normalised P-I curve for elastic-plastic-hardening structural members. Shi et al. [16] used the FEM software package LSDYNA in an attempt to derive empirical expressions for minimum impulse and minimum peak reflected pressure asymptotes to be used to calculate individual P-I curves from a normalised PI curve for RC columns under external free air blasts. Huang et al. [17] investigated the resistance-deflection $(R \Delta)$ function and its effects on PI curves. It was found that, for an SDOF system, the shape of the $R \Delta$ function also had influence over the normalised PI curve. Recently Dragos et al. [18] used a finite difference model to develop normalised P-I curves for RC beams under external free air blasts.

These previous studies conducted on normalised P-I curves have only investigated pulse shapes of pressure-time histories corresponding to blasts in an unconfined environment. However, as noted previously, blasts can also occur in confined environments in which shockwave reflections off surrounding walls and long duration gas pressures can occur. This can cause pressure-time histories with irregular pulse shapes to act on surrounding structural members. However, little research has been carried out to study the effects of blasts with irregular pulse shapes on P-I curves for structural members. Since blasts in a confined environment can be simplified as a bilinear pulse shape [9], a thorough understanding of the effects of bilinear pulse shape on the normalised P-I curve of structural members with bilinear $R \Delta$ functions is required. 
In this paper, the SDOF approach is used to develop analytical solutions for bilinear, elastic plastic hardening, members under bilinear blast loads. These analytical solutions are then used for the derivation of normalised P-I diagrams for bilinear members under bilinear blast loads.

A parametric study was then undertaken to, separately, investigate the effects of the shape of the bilinear pulse load and the bilinear $R \Delta$ function on the normalised P-I curve. A comparison was then undertaken to determine whether the simple approach provided by Li \& Meng [12] for eliminating pulse shape effects on elastic normalised P-I curves could be replicated for eliminating pulse shape effects for bilinear pulse loads. Furthermore, it was also investigated whether, for a given pulse load shape, it was possible to eliminate the effects of the shape of the $R \Delta$ function on the normalised P-I curve.

\section{SDOF MODEL}

SDOF models have widely been adopted by current guidelines in the prediction of the response of a member that is subjected to a dynamic load, such as a blast load. This model provides a representation of the deflection of an important point on the member, such as the midpoint [19]. The deflection of the member over time under blasts can be described by the following differential equation:

$$
M \ddot{y}+R(y)=F(t)
$$

Where $t$ is time, $y$ is the member's deflection, $\ddot{y}$ is the member's acceleration, $M$ is the equivalent mass of the member, $R(y)$ is the $R \Delta$ function and $F(t)$ is the dynamic force on the member over time. It should be noted that the damping coefficient is neglected for blast analysis.

The SDOF model will be developed based on the bilinear load function seen in Figure 3. As the function is bilinear, this means that $F(t)$, seen in eqn (1), will be a two-step piece-wise linear function.

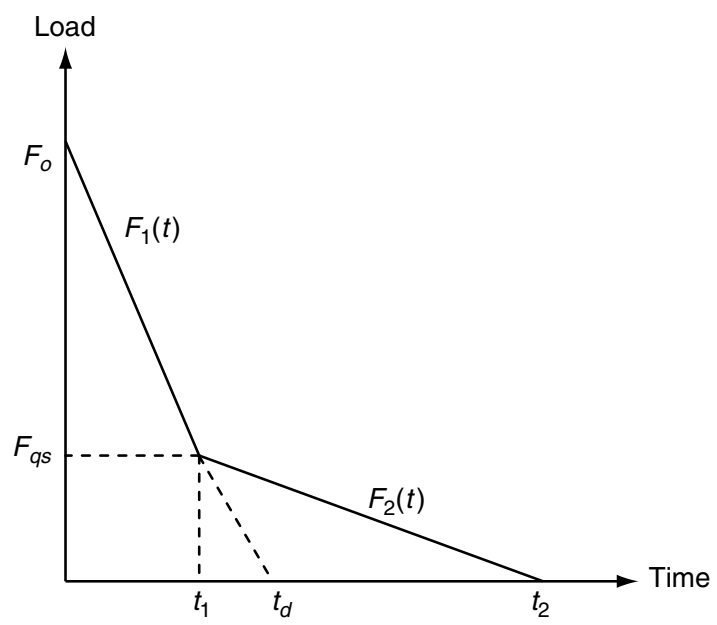

Figure 3. Load function for a simplified confined blast 
The shape of the load function in Figure 3 can be easily manipulated via two parameters which are defined in eqns (2-3). These parameters, $\rho$ and $\tau$, are both functions of the parameters within Figure 3.

$$
\begin{gathered}
\rho=\frac{F_{q s}}{F_{o}} \\
\tau=\frac{t_{1}}{t_{2}}
\end{gathered}
$$

The SDOF model will be developed based on the bilinear R $\Delta$ function seen in Figure 4. As the function is bilinear, this means that $R(\mathrm{y})$, seen in eqn (1), will also be a two-step piecewise linear function.

Similar to the load function, the shape of the $R \Delta$ function in Figure 4 can also be easily manipulated via two parameters which are defined in eqns (4-5). These parameters, $\alpha$ and $\beta$, are both functions of the parameters in Figure 4.

$$
\begin{gathered}
\alpha=\frac{R_{y}}{R_{u}} \\
\beta=\frac{y_{y}}{y_{m}}
\end{gathered}
$$

A typical P-I diagram, such as in Figure 5, contains a P-I curve which is the boundary between all the combinations of peak reflected pressure $\left(P_{\mathrm{r}}\right)$ and impulse $(I)$ of a given blast load which causes a member to fail or survive. As shown in Figure 5, a P-I curve contains three regions: Impulse controlled, Dynamic and Quasi-Static. For an impulse controlled blast,

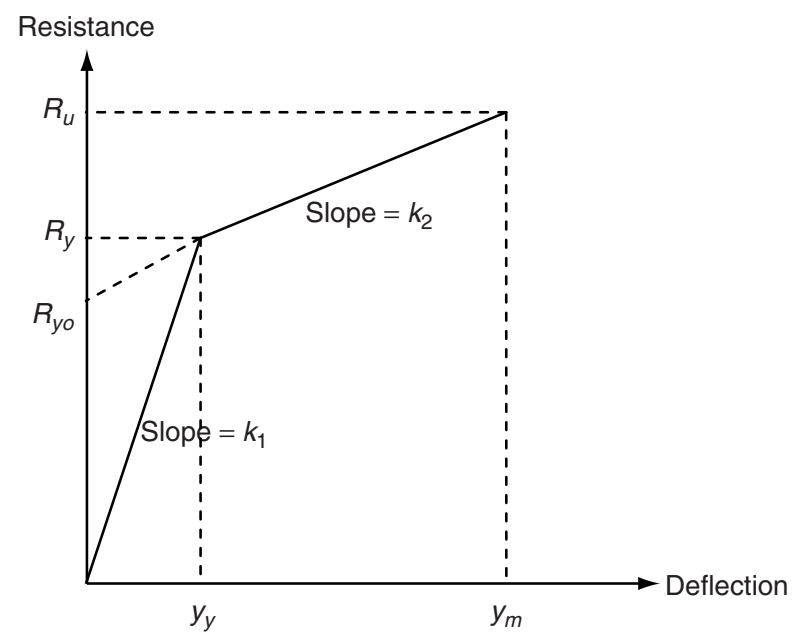

Figure 4. Bilinear Rs function 


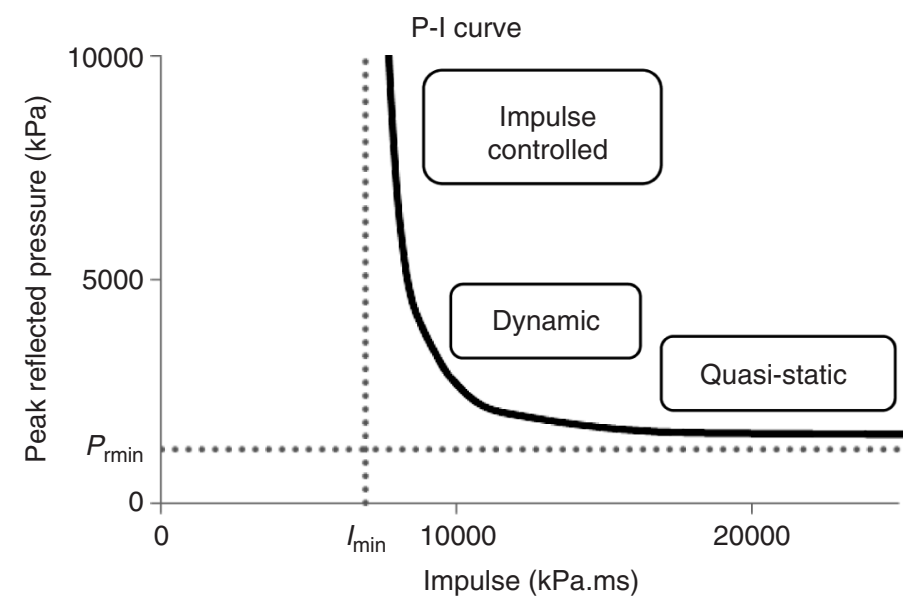

Figure 5. Typical pressure-impulse diagram

the load dissipates before the structural member has time to deflect or respond and therefore only the impulse of the load determines the response of the member. For the quasi-static blast, the structural member responds well before the load has ended and therefore the peak reflected pressure determines the response of the member. In the dynamic region, the duration of the load is comparable to the time at which the maximum deflection, defined as $t_{\max }$, occurs and therefore both the peak reflected pressure, impulse and the shape of the load function all influence the member response. The SDOF model will be used to determine points within this region of the P-I curve, as this is the region which is influenced by the shape of pulse load, $\rho$ and $\tau$, and the shape of the $R \Delta$ function, $\alpha$ and $\beta$. This will be done by introducing a new dimensionless parameter $\eta$, which is the ratio of $t_{\max }$ to $t_{2}$, where $t_{\max }$ is the time at which the member reaches its maximum deflection, such that $y=y_{m}$ and $y^{\prime}=0$, and $t_{2}$ is the total duration of the pressure-time history as seen in Figure 3. By choosing a specific value for $\eta$, a new equation is added to the final conditions, which ensures that a specific point on the P-I curve is obtained. Furthermore, by letting $\eta$ equal 1 , or slightly more or less than 1 , ensures that the duration of the load, $t_{2}$, is comparable to the time at which the maximum deflection occurs, $t_{\max }$, which is how a point in the dynamic region of the P-I curve is defined.

In addition to the P-I curve, Figure 5 also displays the horizontal and vertical asymptotes to which the P-I curve tends at each extreme, shown as the dotted lines. The horizontal asymptote, $P_{\text {rmin }}$, is the minimum peak reflected pressure asymptote whereas the vertical asymptote, $I_{\min }$, is the minimum impulse asymptote. For any SDOF system, $P_{\text {rmin }}$ and $I_{\min }$ can be determined using eqns (6-7), respectively, which are derived based on the laws of conservation of energy.

$$
\begin{gathered}
P_{\text {rmin }}=\frac{E_{R}}{y_{m}} \\
I_{\text {min }}=\sqrt{2 M E_{R}}
\end{gathered}
$$

Where $y_{m}$ is the maximum deflection at which failure occurs, $M$ is the equivalent mass of the member and $E_{R}$ is the area under the $R \Delta$ function from 0 to $y_{m}$. 
After the SDOF model is used to determine the coordinates of a point on the P-I curve, the $I$ and $P_{r}$ coordinates will be divided by $I_{\min }$ and $P_{\text {rmin }}$, respectively, to determine the coordinates of that point on the normalised P-I curve. This is because the normalised P-I curve corresponds to a P-I curve in which the asymptotes, $I_{\min }$ and $P_{\text {rmin }}$, are unity.

\section{DYNAMIC ANALYSIS}

In order to undertake the dynamic analysis, as both the load and $R \Delta$ functions are bilinear, there are four cases that need to be solved for and each case has its governing differential equation of motion. These are when:

1) Both the bilinear load and $R \Delta$ functions are in the first stage (Elastic, $F_{1}(t)$ ).

2) The load function is still in its first stage but the $R \Delta$ function has progressed into the plastic region (Plastic, $F_{1}(t)$ ).

3) The load function is in the second stage while the $R \Delta$ function is still in the elastic region (Elastic, $F_{2}(t)$ ).

4) Both the load and $R \Delta$ functions are in the second stage (Plastic, $F_{2}(t)$ ).

For any given blast load, only three of the above cases need to be taken into consideration. This depends on how the changeover between the two functions occurs, thus whether $y_{\mathrm{y}}$ or $F_{q s}$ is reached first. Consequently, the blast load and structural response interaction either progresses from Case 1 to Case 2 then Case 4, thus $y_{y}$ is reached first. Conversely, the interaction may progress from Case 1 to Case 3 then Case 4 , thus $F_{q s}$ is reached first. Since there are different load and $R \Delta$ functions to consider for each of the four cases, there are four differential equations to describe the member's motion:

$$
\begin{gathered}
m \ddot{y}+k_{1} y=F_{1}(t) \\
m \ddot{y}+R_{y o}+k_{2}(y)=F_{1}(t) \\
m \ddot{y}+k_{1} y=F_{2}(t) \\
m \ddot{y}+R_{y o}+k_{2}(y)=F_{2}(t)
\end{gathered}
$$

Where $F_{1}(t)$ and $F_{2}(t)$ describe the force on the member, seen in Figure $3, R_{y o}$ is the resistance if the plastic branch of the $R \Delta$ function is extrapolated to a deflection of 0 and $k_{1}$ and $k_{2}$ are the stiffnesses of the first and second branches, respectively, of the $R \Delta$ function, all seen in Figure 4.

The first progression that was considered was when the $R \Delta$ function changes from the elastic to plastic region before the load function changes from its first to its second region. Therefore, the progression is Case 1 to Case 2 then Case 4. For this situation, the blast load progresses through Case 1 until the deflection is equal to $y_{y}$, then Case 2 until $t_{1}$ is reached, and finally Case 4 until the time when the maximum deflection is reached.

For Case 1, the differential equation that needs to be solved to obtain the deflection is eqn (8). This equation is solved based on the initial conditions: $y(0)=0, y^{\prime}(0)=0$.

In a similar way to an elastic problem, the equation proposed is used to describe the motion for this first Case. This can be seen by

$$
y=\frac{F_{o}}{k_{1}}\left(1-\cos \left(\omega_{1} t\right)\right)+\frac{F_{o}}{k_{1} t_{d}}\left(\frac{\sin \left(\omega_{1} t\right)}{\omega_{1}}-t\right)
$$




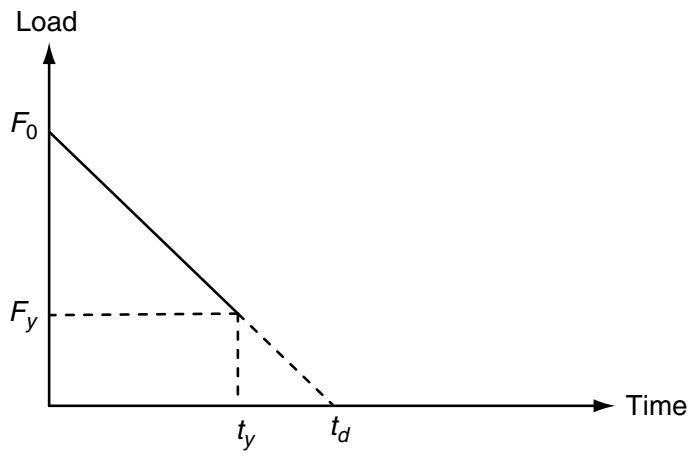

Figure 6. Pressure-time history for the first phase of the blast

In eqn (12), $t_{d}$ is the time at which, if the first branch of the load function was extrapolated, the load reduces to zero, as seen in Figure 6, and $\omega_{1}$ is the natural frequency of the member during its elastic phase. Since it is assumed that the blast shape is triangular, and the first section of the blast does not reduce to zero, $t_{d}$ was found using similar triangles. In Figure $6, t_{y}$ is the time at which yield deflection $\left(y_{y}\right)$ is reached.

The value that is found for $t_{d}$ is given by

$$
t_{d}=\frac{F_{o} t_{y}}{F_{o}-F_{y}}
$$

Where $F_{y}$ is the load caused by the blast at the time $t_{y}$. Therefore, if this final time, $t_{d}$, is substituted into eqn (13), the deflection until $t_{y}$ can be described by

$$
y=\frac{F_{o}}{k_{1}}\left(1-\cos \left(\omega_{1} t\right)\right)+\frac{\left(F_{o}-F_{y}\right)}{k_{1} t_{y}}\left(\frac{\sin \left(\omega_{1} t\right)}{\omega_{1}}-t\right)
$$

For Case 2, eqn (9) is solved based on the initial conditions shown in eqns (15-18), which in the previous case, are the conditions corresponding to time $t_{y}$. This is done such that $t_{y}$ now corresponds to $t=0$ in this new case.

$$
\begin{gathered}
y(0)=y_{y} \\
y^{\prime}(0)=y_{y}^{\prime} \\
F(0)=F_{y} \\
F\left(t_{1}-t_{y}\right)=F_{q s}
\end{gathered}
$$


Since there are particular and homogeneous solutions when solving eqn (9), the deflection for this second case can be described by

$$
y=c_{1,1} \cos \left(\omega_{2} t\right)+c_{2,1} \sin \left(\omega_{2} t\right)+A_{1} t+B_{1}
$$

Using the initial conditions mentioned previously, the constants $\left(c_{1,1}, c_{2,1}, A_{1}\right.$ and $\left.B_{1}\right)$ within eqn (19) can be determined:

$$
\begin{gathered}
c_{1,1}=y_{y}+\frac{R_{y o}-R_{y}}{k_{2}} \\
c_{2,1}=y_{y}+\frac{R_{y}-F_{q s}+k_{2} y_{y}^{\prime}\left(t_{1}-t_{y}\right)}{k_{2} \omega_{2}\left(t_{1}-t_{y}\right)} \\
A_{1}=\frac{F_{q s}-R_{y}}{k_{2}\left(t_{1}-t_{y}\right)} \\
B_{1}=\frac{R_{y}-R_{y o}}{k_{2}}
\end{gathered}
$$

Now that the deflection up to time $t_{1}$ is known, Case 4 can be solved to determine the deflection from $t_{1}$ until the maximum deflection. For this case, eqn (11) can be solved based on the initial conditions shown in eqns (24-27), which in the previous case, are the conditions corresponding to time $t_{1}$. This is done such that $t_{1}$ now corresponds to $t=0$ in this new case.

$$
\begin{gathered}
y(0)=y_{1} \\
y^{\prime}(0)=y_{1}^{\prime} \\
y(0)=F_{q s} \\
F\left(t_{2}-t_{1}\right)=0
\end{gathered}
$$

Similar to the deflection for Case 2, solving for the deflection for Case 4 requires finding the particular and homogeneous solution to the equation. This can be seen in eqn (28).

$$
y=c_{3,1} \cos \left(\omega_{2} t\right)+c_{4,1} \sin \left(\omega_{2} t\right)+C_{1} t+D_{1}
$$

Using the initial conditions, the constants in eqn (44) $\left(c_{3,1}, c_{4,1}, C_{1}\right.$ and $\left.D_{1}\right)$ can be solved for, to become: 


$$
\begin{gathered}
c_{3,1}=y_{1}-\frac{F_{q s}-R_{y o}}{k_{2}} \\
c_{4,1}=\frac{c_{3,1} \sin \left(\omega_{2}\left(\eta t_{2}-t_{1}\right)\right)-\frac{y_{1}^{\prime} / \omega_{2}}{\cos \left(\omega_{2}\left(\eta t_{2}-t_{1}\right)\right)-1}}{C_{1}=y_{1}^{\prime}-\omega_{2} c_{4,1}} \\
D_{1}=\frac{F_{q s}-R_{y o}}{k_{2}}
\end{gathered}
$$

Therefore, the deflection can now be found when the blast progresses through Case 1 to Case 2 then Case 4. The second progression considered corresponds to when the load function changes from its first to its second phase before the $R \Delta$ function changes from the elastic to plastic region. Therefore, the progression is Case 1 to Case 3 then Case 4 . For this progression, the blast progresses through Case 1 until $t_{1}$, then Case 3 until the deflection $y_{\mathrm{y}}$ is reached, and finally Case 4 until the time when the maximum deflection is reached.

For Case 1, the differential equation that needs to be solved to obtain the deflection is eqn (8). This equation is solved based on the initial conditions: $y(0)=0, y^{\prime}(0)=0$.

Similar to the previous progression considered, the equation is used to describe the motion for this first case. This can be seen in eqn (33).

$$
y=\frac{F_{o}}{k_{1}}\left(1-\cos \left(\omega_{1} t\right)\right)+\frac{F_{o}}{k_{1} t_{d}}\left(\frac{\sin \left(\omega_{1} t\right)}{\omega_{1}}-t\right)
$$

In eqn (33), $t_{d}$ is the time at which, when extrapolated, the first branch of the load function reduces to zero, as seen in Figure 3. Since it is assumed that the blast shape is triangular, and the first section of the blast does not reduce to zero, $t_{d}$ was found using similar triangles and can be seen in eqn (34).

$$
t_{d}=\frac{F_{o} t_{1}}{F_{o}-F_{q s}}
$$

Therefore, if $t_{d}$ is substituted into eqn (33), the deflection until $t_{1}$ can be described by eqn (35).

$$
y=\frac{F_{o}}{k_{1}}\left(1-\cos \left(\omega_{1} t\right)\right)+\frac{\left(F_{o}-F_{q s}\right)}{k_{1} t_{1}}\left(\frac{\sin \left(\omega_{1} t\right)}{\omega_{1}}-t\right)
$$

For Case 3, eqn (10) is solved based on the initial conditions shown in eqns (36-39) which are the conditions at time $t_{1}$ in the previous case. This is done such that $t_{1}$ now corresponds to $t=0$ in this new case. 


$$
\begin{gathered}
y(0)=y_{1} \\
y^{\prime}(0)=y_{1}^{\prime} \\
F(0)=F_{q s} \\
F\left(t_{y}-t_{1}\right)=R_{y}
\end{gathered}
$$

Since there are also particular and homogeneous solutions when solving eqn (10), the deflection for this case can be described by

$$
y=c_{1,2} \cos (\omega t)+c_{2,2} \sin (\omega t)+A_{2} t+B_{2}
$$

Using the initial conditions described above, the constants $\left(c_{1,2}, c_{2,2}, A_{2}\right.$ and $\left.B_{2}\right)$ within eqn (40) can be determined.

$$
\begin{gathered}
c_{1,2}=y_{1}-\frac{F_{q s}}{k_{1}} \\
c_{2,2}=\frac{F_{q s}-R_{y}+k_{1} y_{2}^{\prime}\left(t_{y}-t_{1}\right)}{k_{1} \omega_{1}\left(t_{y}-t_{1}\right)} \\
A_{2}=\frac{R_{y}-F_{q s}}{k_{1}\left(t_{y}-t_{1}\right)} \\
B_{2}=\frac{F_{q s}}{k_{1}}
\end{gathered}
$$

Since the deflection until $t_{y}$ is known, Case 4 can now be solved to determine the deflection from $t_{y}$ until the maximum deflection. For this case, eqn (11) can be solved based on the initial conditions shown in eqns (45-48) which are the conditions at time $t_{y}$ in the previous case. This is done based on the assumption that $t_{y}$ now corresponds to time $t=0$.

$$
\begin{aligned}
& y(0)=y_{y} \\
& y^{\prime}(0)=y_{y}^{\prime} \\
& F(0)=R_{y}
\end{aligned}
$$




$$
F\left(t_{2}-t_{y}\right)=0
$$

Similar to the deflection for Case 3, solving for the deflection for Case 4 requires finding the particular and homogeneous solutions. This is shown in eqn (49).

$$
y=c_{3,2} \cos \left(\omega_{2} t\right)+c_{4,2} \sin \left(\omega_{2} t\right)+C_{2} t+D_{2}
$$

Using the initial conditions, the constants within eqn (49) $\left(c_{3,2}, c_{4,2}, C_{2}\right.$ and $\left.D_{2}\right)$ can be solved for, to become:

$$
\begin{gathered}
c_{3,2}=y_{y}-\frac{R_{y}-R_{y o}}{k_{2}} \\
c_{4,2}=\frac{c_{3,2} \sin \left(\omega_{2}\left(\eta t_{2}-t_{y}\right)\right)-\frac{y_{y}^{\prime} / \omega_{2}}{\cos \left(\omega_{2}\left(\eta t_{2}-t_{y}\right)\right)-1}}{C_{2}=y_{y}^{\prime}-\omega_{2} c_{4,2}} \\
D_{2}=\frac{R_{y}-R_{y o}}{k_{2}}
\end{gathered}
$$

Therefore, the deflection can now be found if the blast progresses through Case 1 to Case 3 then Case 4.

As a result, whatever the progression of the blast, the deflection can now be found. Consequently, the peak pressure, $P_{r}$, and impulse, $I$, can be manipulated such that at time $=\eta t_{2}$, $y=y_{m}$ and $y^{\prime}=0$. These final conditions ensure that the maximum deflection, with a velocity of 0 , occur at time $\eta t_{2}$. By doing this, it means that $\eta$ can be manipulated in order to determine the coordinates of more than one point within the dynamic region of the PI curve. Furthermore, eqns (2-3) can then be used to convert these coordinates from that of a point on the P-I curve to that of a point on the normalised P-I curve. For example, as four different values for $\eta$ are being considered, 0.7, 0.8, 0.9 and 1, four points within the dynamic region of the normalised P-I diagram can be generated. As these four points on the normalised P-I curve and both the horizontal and vertical asymptotes are known, this data can be used to determine a suitable function for the entire normalised P-I curve. This can then be repeated for different bilinear pulse shape parameters, $\rho$ and $\tau$, and different $\mathrm{R} \Delta$ function shape parameters, $\alpha$ and $\beta$, in order to determine many normalised P-I curves. An example of these points was found for the bilinear pulse shape parameters $\rho$ $=0.2$ and $\tau=0.2$ and $R \Delta$ function shape parameters $\alpha=0.95$ and $\beta=0.1$ and can be seen in Figure 7. 


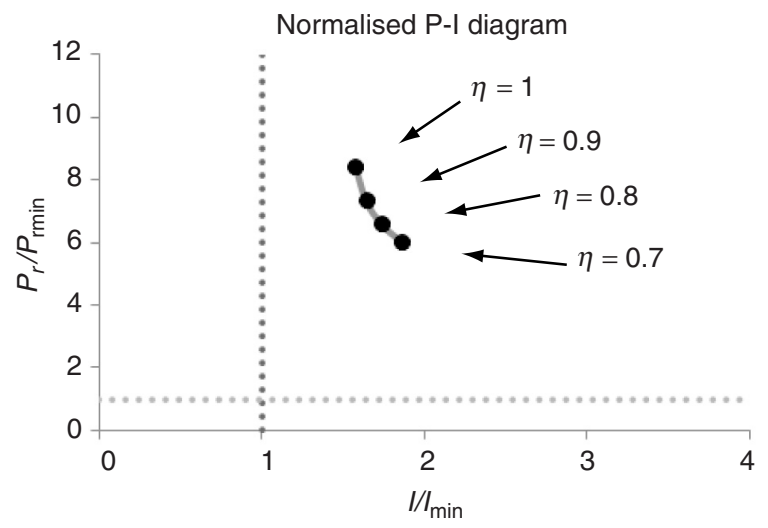

Figure 7. Points generated on the normalised P-I diagram when $\rho=0.1$, $\tau=0.15, \alpha=0.95, \beta=0.1$

\section{PARAMETRIC STUDIES}

Parametric studies were conducted to investigate how the shape parameters of the load and $R \Delta$ functions affect the shape of the normalised P-I curve. In order to discover some kind of correlation or pattern, the shape parameters for the load function and $R \Delta$ function were altered separately. Therefore, first the effects of the pulse load shape parameters, $\rho$ and $\tau$, on the normalised P-I curve were investigated for a typical $R \Delta$ function. Then, the effects of the $R \Delta$ function shape parameters, $\alpha$ and $\beta$, on the normalised P-I curve for a given pulse load shape were studied.

In order to obtain an entire normalised P-I curve from the four determined points of a normalised P-I curve, a function provided by Li \& Meng [12] was employed and can be seen in eqn (54).

$$
p=\frac{n_{1}}{(i-1)^{n 2}}+0.5
$$

However, since each of the asymptotes of the normalised P-I curve are 1 in this case, eqn (54) needs to be modified to the form shown in eqn (55) to account for this.

$$
p=\frac{n_{1}}{(i-1)^{n 2}}+1
$$

It should be noted that as eqn (55) represents an equation for the normalised P-I curve, $p=P_{r} / P_{\text {rmin }}$ and $i=I / I_{\min }$. Eqn (55) also contains two coefficients, $n_{1}$ and $n_{2}$. These two coefficients can be manipulated to alter the shape of the dynamic region of the normalised P-I curve. Therefore, by knowing the $I / I_{\min }$ and $P_{r} / P_{\text {rmin }}$ values for each of the four points of a given normalised P-I curve corresponding to given pulse load shape and $R \Delta$ function shape parameters, $n_{1}$ and $n_{2}$ values were found such that the difference between the actual coordinates and that found using eqn (55) was minimised. This was then repeated to find $n_{1}$ and $n_{2}$ values corresponding to normalised P-I curves for many pulse load shape and $R \Delta$ function shape parameters. 


\section{NORMALISED P-I DIAGRAMS FOR BILINEAR BLAST LOADS}

As a partially confined blast can be approximated by a bilinear pulse load, a series of normalised P-I curves were generated to cover as great a range of bilinear pulse loads as possible. Using the SDOF model and eqn (55), normalised P-I diagrams were created for all combinations of $\rho=0.1,0.2,0.4,0.6$ and $0.8, \tau=0.1,0.2,0.4,0.6$ and 0.8 . Exceptions to this were when $(\rho+\tau)>1$, because such pulse shapes would not be possible in reality. For the condition when $\rho+\tau=1$, a straight line is formed, and hence normalised P-I curves for combinations under this condition could be duplicated.

Two examples of normalised P-I curves can be seen in Figure 8, where the four points are displayed along with the curve that was fitted to those points using eqn (55). Figure 8(a) corresponds to a normalised P-I curve of a member subjected to a bilinear blast pulse shape with $\rho=0.1$ and $\tau=0.6$. Figure 8 (b) corresponds to a normalised P-I curve of a member subjected to a bilinear blast pulse shape with $\rho=0.2$ and $\tau=0.2$. Normalised P-I curves corresponding to other bilinear pulse shapes that were generated can be seen in Figure 9. All normalised P-I curves seen in Figures 8 and 9 correspond to a member with $R \Delta$ function shape parameters of $\alpha=0.7$ and $\beta=0.3$.

It can be seen from Figure 9 that varying the bilinear pulse shape parameters has a significant impact on the shape of the normalised P-I curve. All of the cases considered display similar patterns, with variations only occurring in the dynamic region of the curve. The $n_{1}$ and $n_{2}$ values that were obtained to fit the different curves can be seen in Tables 1 and 2, respectively. When generating normalised P-I curves, for some combinations the solver program was not able to accurately generate the coordinates of a point on the normalised P-I curve. Consequently, the combinations that did not generate points are left blank in the tables.

These $n_{1}$ and $n_{2}$ values, seen in Tables 1 and 2, respectively, were then plotted against $\tau$, for varying $\rho$ values. The plot of $n_{1}$ and $n_{2}$ values can be seen in Figures 10(a) and 10(b), respectively.

Figures 10(a) and 10(b) illustrate that the interaction between the $n$ coefficients and the pulse shape parameters, $\rho$ and $\tau$, is quite complex. Due to the complexity within the correlations, it would be very difficult to determine an equation, or set of equations, to relate $\rho$ and $\tau$ to $n_{1}$ and $n_{2}$.

(a)

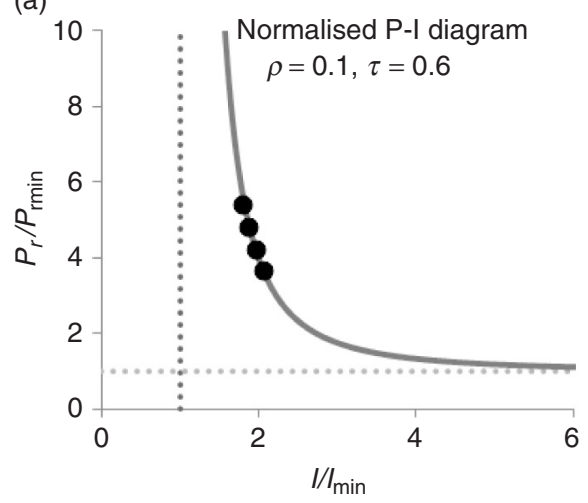

(b)

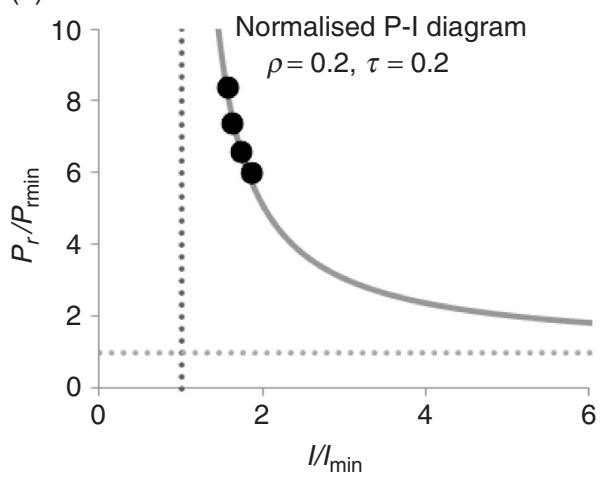

Figure 8. Normalised P-I curves generated for two different bilinear pulse shapes 


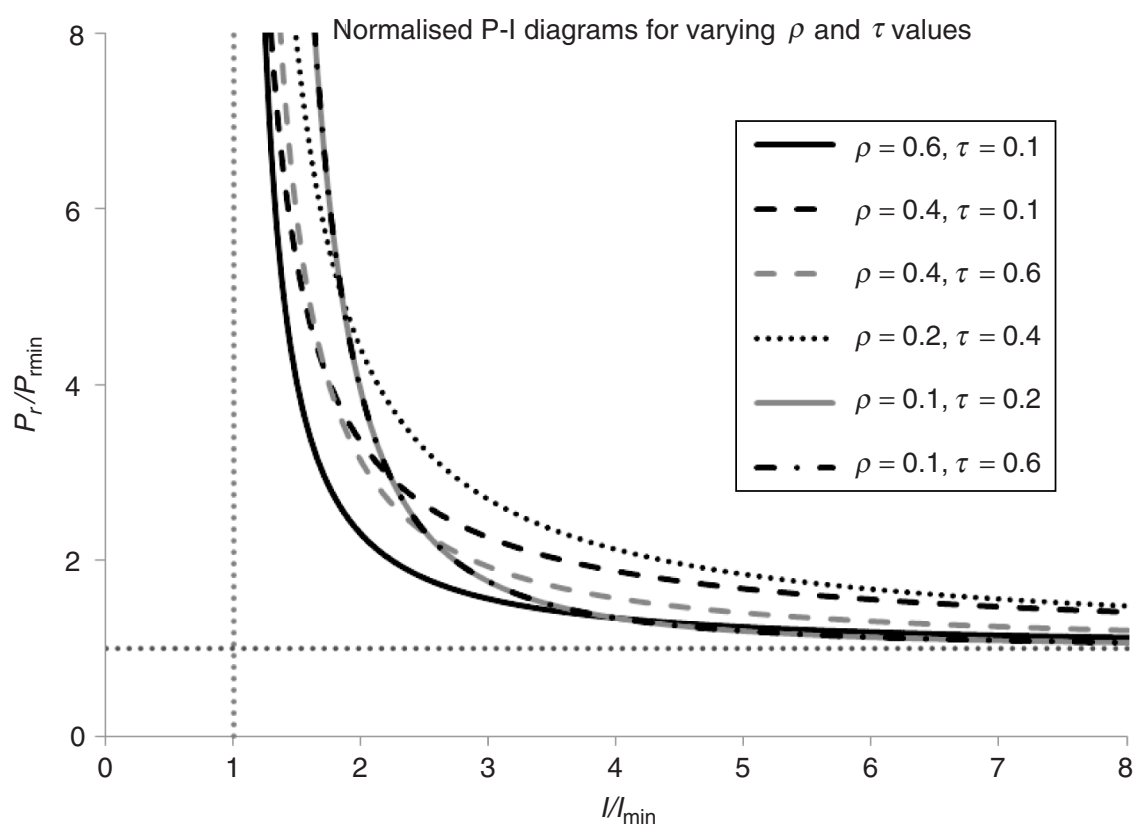

Figure 9. Pressure-impulse curves for varying $\rho$ and $\tau$ values ( $\alpha=0.7 \&$ $\beta=0.3$ )

Table 1. $n_{1}$ values for varying $\rho$ and $\tau$ values ( $\alpha=0.7 \& \beta=0.3$ )

\begin{tabular}{llcccc} 
& & \multicolumn{3}{c}{$\boldsymbol{\rho}$} \\
\cline { 3 - 6 } $\boldsymbol{n}_{\mathbf{1}}$ & & $\mathbf{0 . 1}$ & $\mathbf{0 . 2}$ & $\mathbf{0 . 4}$ & $\mathbf{0 . 6}$ \\
\hline $\boldsymbol{\tau}$ & 0.1 & & & 2.350 & 1.300 \\
\hline & 0.2 & 2.950 & 1.848 & 2.400 & \\
\hline & 0.4 & 3.850 & 3.423 & 2.575 & \\
\hline & 0.6 & 2.950 & 2.800 & 2.150 & \\
\hline
\end{tabular}

Table 2. $n_{2}$ values for varying $\rho$ and $\tau$ values $(\alpha=0.7 \& \beta=0.3$ )

\begin{tabular}{llcccc} 
& & \multicolumn{3}{c}{$\boldsymbol{\rho}$} \\
\cline { 3 - 6 } $\boldsymbol{n}_{\mathbf{2}}$ & & $\mathbf{0 . 1}$ & $\mathbf{0 . 2}$ & $\mathbf{0 . 4}$ & $\mathbf{0 . 6}$ \\
\hline $\boldsymbol{\tau}$ & 0.1 & & & 0.900 & 1.200 \\
\hline & 0.2 & 1.950 & 0.449 & 0.950 & \\
\hline & 0.4 & 0.988 & 1.015 & 1.000 & \\
\hline 0.6 & 1.950 & 1.400 & 1.200 & \\
\hline
\end{tabular}

According to Li \& Meng [12], the pulse shape effects for elastic normalised P-I curves can be eliminated via eqn (54) or eqn (55). They state that the pulse shape parameter, $d$, seen in eqn (56), can be calculated for a given pulse load shape, which can then be used 
(a)

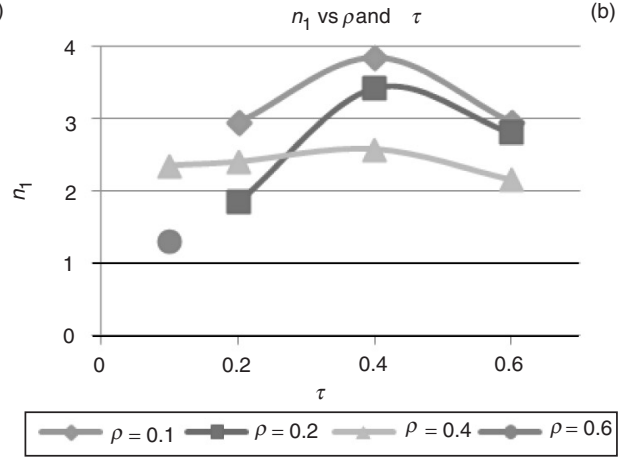

(b)

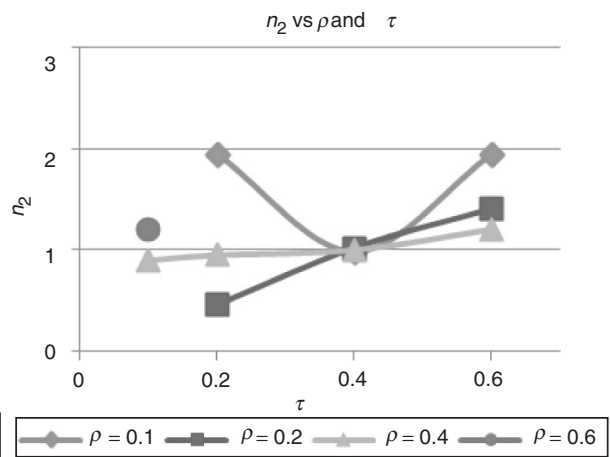

Figure 10. Plot of $n_{1}(\mathrm{a})$ and $n_{2}$ (b) values for varying $\rho$ and $\tau$ values $(\alpha=0.7 \& \beta=0.3)$

to calculate $n_{1}$ and $n_{2}$ to determine the appropriate shape of the corresponding normalised P-I curve.

$$
d=\sqrt{x_{0}^{2}+y_{0}^{2}}
$$

Where $x_{0}$ and $y_{0}$ are the centroids along the time and force axes, respectively, for a pulse load which has both a peak load and a total duration of 1 .

Although for this case, a bilinear elastic-plastic-hardening member is being solved for where $\alpha=0.7 \& \beta=0.3$ instead of an elastic member, the aim is to establish whether a correlation can be made between the $n$ coefficients and the $d$ values for each bilinear pulse load shape. For each of the 11 bilinear pulse load shapes, for which $n_{1}$ and $n_{2}$ coefficients have been determined in Tables 1 and 3, respectively, their corresponding $d$ values have been calculated. From this, $n_{1}$ and $n_{2}$ values were plot against their corresponding $d$ values and can be seen within Figures 11(a) and 11(b), respectively.

Li \& Meng [12] showed that for exponential, rectangular and triangular pulse load shapes, typically associated with external blasts, the $d$ value can be successfully used to calculate $n_{1}$ and $n_{2}$, which can then be used to determine an accurate normalised P-I curve for that pulse

(a)

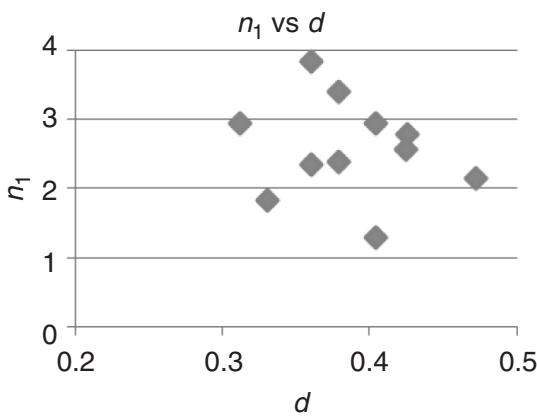

(b)

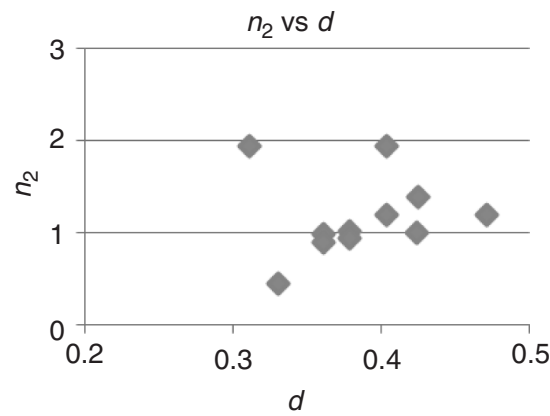

Figure 11. Plot of $n_{1}(a)$ and $n_{2}$ (b) values for bilinear pulse load shapes with varying $d$ values $(\alpha=0.7 \& \beta=0.3$ ) 
load shape. This was only possible because a direct correlation could be made between $d$ and both $n_{1}$ and $n_{2}$. However, due to the scatter of the plots, observed in both Figures 11(a) and 11(b), it can be seen that no correlation exists between the $d$ values and both $n_{1}$ and $n_{2}$ for bilinear pulse load shapes. Although Li \& Meng [12] was able to use eqn (54) to eliminate pulse shape effects for elastic normalised P-I curves for external blasts, it can be seen that this approach cannot be applied to bilinear pulse load shapes associated with partially confined blasts. However, by conducting a complete parametric study, a series of tables, such as Tables 1 and 2, can be developed which will allow a normalised P-I curve corresponding to any bilinear pulse shape to be estimated.

\section{NORMALISED P-I DIAGRAMS FOR BILINEAR RD FUNCTIONS}

An investigation into the effects of the shape of the RA function on the shape of the normalised P-I curve was then undertaken. Points on the normalised P-I curves were obtained for various $R \Delta$ function shapes by keeping the pulse load shape parameters constant, such that $\rho=0.4$ and $\tau=0.4$. Similarly to when the blast parameters were altered, curves corresponding to eqn (55) were then fitted to these points to generate the normalised $\mathrm{P}$-I curves for varying $R \Delta$ functions, as seen in Figure 12. These normalised P-I curves were then compared to examine the influence of $\alpha$ and $\beta$, and thus $R \Delta$ function shape, on the shape of the normalised P-I curve. The $n_{1}$ and $n_{2}$ values obtained to form these normalised P-I curves can be seen in Tables 3 and 4, respectively.

From Figure 12, it can be seen that varying $\alpha$ and $\beta$ has a significant effect on the normalised P-I curves produced. Table 3 shows that as $\beta$ decreases, and thus ductility

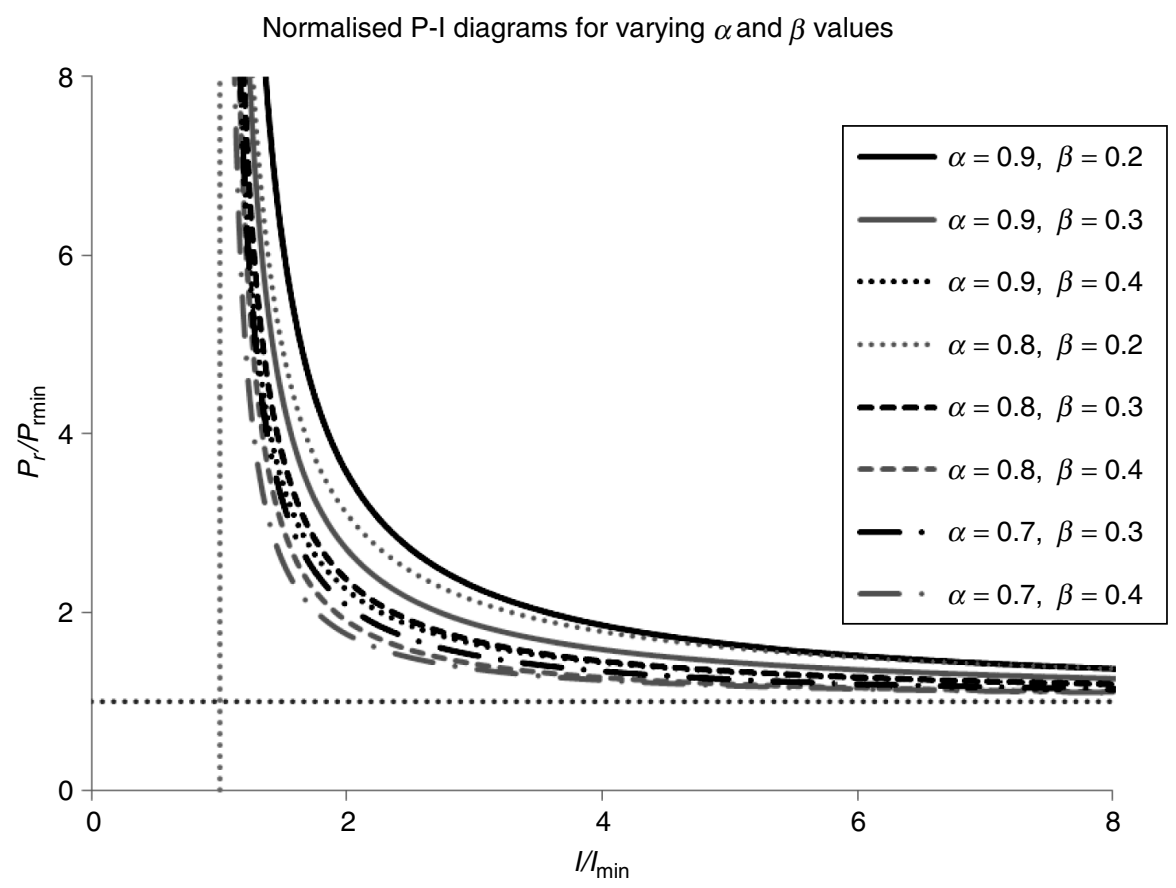

Figure 12. Normalised P-I curves for varying bilinear R $\Delta$ function shape parameters $(\rho=0.4$ and $\tau=0.4)$ 
Table 3. $n_{1}$ values for varying $\alpha$ and $\beta$ values ( $\rho=0.4$ and $\tau=0.4$ )

\begin{tabular}{ccccc} 
& & \multicolumn{1}{c}{$\boldsymbol{\alpha}$} \\
\cline { 3 - 5 } $\boldsymbol{n}_{\mathbf{1}}$ & & $\mathbf{0 . 7}$ & $\mathbf{0 . 8}$ & $\mathbf{0 . 9}$ \\
\hline $\boldsymbol{\beta}$ & 0.15 & & & 3.250 \\
\hline & & 2.100 & 2.575 \\
\hline & 0.2 & 1.080 & 1.368 & 1.700 \\
\hline & 0.3 & 0.750 & 0.900 & 1.250 \\
\hline
\end{tabular}

Table 4. $n_{2}$ values for varying $\alpha$ and $\beta$ values ( $\rho=0.4$ and $\tau=0.4$ )

\begin{tabular}{ccccc} 
& & \multicolumn{2}{c}{} \\
\cline { 3 - 5 } $\boldsymbol{n}_{\mathbf{2}}$ & $\mathbf{0 . 7}$ & $\mathbf{0 . 8}$ & $\mathbf{0 . 9}$ \\
\hline $\boldsymbol{\beta}$ & 0.15 & & & 0.950 \\
\hline & & 0.900 & 1.000 \\
\hline & & 1.012 & 0.980 \\
\hline & 0.3 & 1.050 & 1.100 & 0.950 \\
\hline
\end{tabular}

increases, $n_{1}$ increases. However, Table 4 shows that the effects of $\alpha$ and $\beta$ on $n_{2}$ are not as significant.

These $n_{1}$ and $n_{2}$ values, seen in Tables 3 and 4, respectively, were then plotted against $\beta$, for varying $\alpha$ values. The plot of $n_{1}$ and $n_{2}$ values can be seen in Figures 13(a) and 13(b), respectively.

Figure 13(a) illustrates a clear correlation between $n_{1}$ and $\beta$ for different $\alpha$ values. Therefore, it would be possible to derive an empirical equation relating $n_{1}$ to the $R \Delta$ function shape parameters, $\alpha$ and $\beta$. Conversely, Figure 13(b) illustrates that little correlation exists between $n_{2}$ and $\alpha$ and $\beta$. However, the figure also shows that $n_{2}$ does not vary significantly. Figure 13 shows that it may be possible to use eqn (54), proposed by Li \& Meng [12], to eliminate the effects of the $R \Delta$ shape parameters. However, this would only apply to specific

(a)

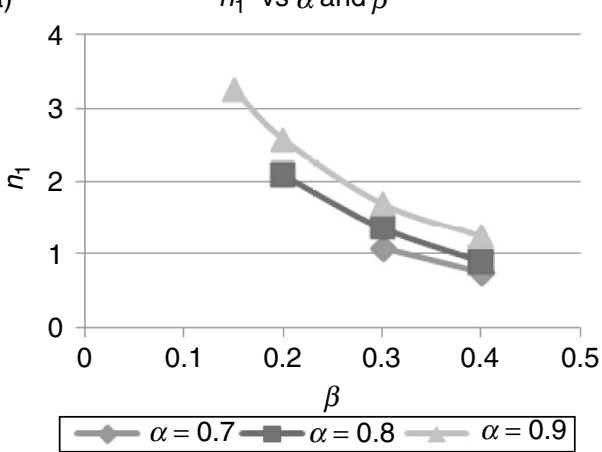

(b)

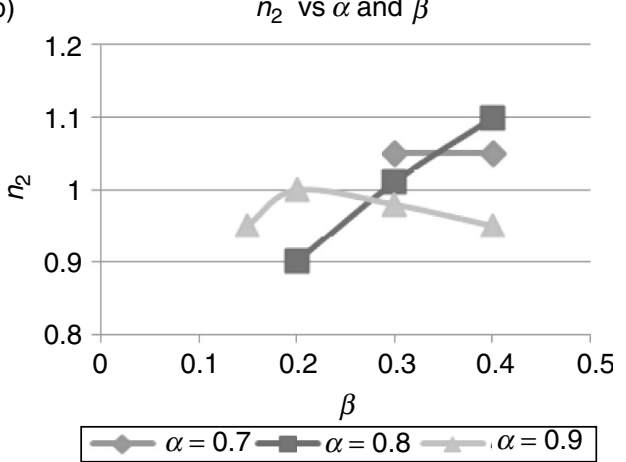

Figure 13. Plot of $n_{1}(a)$ and $n_{2}$ (b) values for varying $\alpha$ and $\beta$ values ( $\rho=0.4$ and $\tau=0.4$ ) 
pulse load shapes. However, by conducting a complete parametric study, a series of tables, similar to Tables 1 - 4, could be developed. These tables could be used to determine $n_{1}$ and $n_{2}$ for a given bilinear pulse shape and given $R \Delta$ function shape in order to estimate the corresponding normalised P-I curve.

\section{CONCLUSION}

SDOF models have been developed to predict the response of members with bilinear, elastic-plastic-hardening, $\mathrm{R} \Delta$ functions under bilinear blast loads typically associated with partially confined blasts. For most cases, the bilinear blast loads provided by UFC guidelines [7] provide a safe estimate. However, Dragos et al. [9] showed that for some scenarios, these estimates provided by UFC guidelines were unconservative and so for such cases, the methods proposed by Dragos et al. [9] can be adopted to determine the corresponding bilinear blast loads. Based on the developed SDOF models, parametric studies are conducted to investigate how the shape parameters of bilinear blasts and bilinear R $\Delta$ functions affect the normalised P-I curve. Then, a series of normalised P-I curves were generated by keeping the shape parameters describing the bilinear pulse load constant or the shape parameters describing the bilinear $\mathrm{R} \Delta$ function constant. The generated normalised P-I curves were then fitted to the equation suggested by Li \& Meng [12], which is proposed to approximate an entire normalised P-I curve. The $n_{1}$ and $n_{2}$ values that were obtained to fit the different curves generated using the equation suggested by Li \& Meng [12] were derived for various bilinear pulse load shapes and various bilinear $\mathrm{R} \Delta$ function shapes. It was then, separately, investigated whether correlations could be made between the $n$ coefficients and the pulse shape parameters and the R $\Delta$ function shape parameters in order to eliminate their effects on the normalised P-I curve. It was discovered that, for bilinear pulse load shapes, no correlation could be found between the pulse shape and the $n$ coefficients. Therefore, the approach cannot be used to eliminate the pulse shape effects of bilinear pulse loads. However, it was discovered that the approach provided by Li \& Meng [12] may be able to eliminate the effects of the shape of the R $\Delta$ function on the normalised P-I curve for a given pulse load shape. It was shown that by conducting a comprehensive parametric study on normalised P-I curves for varying bilinear pulse load shapes and bilinear $R \Delta$ function shapes, a series of tables could be determined which would allow any normalised P-I curve to be estimated for partially confined blasts.

\section{ACKNOWLEDGEMENTS}

The financial and technical support of DSTO and VSL and the financial support of the Australian research Council under ARC Linkage Project LP 0883451 are gratefully acknowledged.

\section{REFERENCES}

[1] Edri, I., Savir, Z., Feldgun, V.R., Karinski, Y.S., \& Yankelevsky, D.Z. (2010). On blast pressure analysis due to a partially confined explosion: 1. Experimental studies. International Journal of Protective Structures, 2 (1), 1-20.

[2] Feldgun,V. R., Kochetkov, A. V., Karinski ,Y. S., Yankelevsky, D. Z. (2008). Internal blast-loading in a buried lined tunnel. International Journal of Impact Engineering, 2008 (35),172-183.

[3] Hu, Y, Wu, C., Lukaszewicz, M., Dragos, J., Ren, J., Haskett, M. (2011). Characteristics of confined blastloading in unvented structures. International Journal of Protective Engineering, 2 (1), 21-43.

[4] Son, J., Lee, H.J. (2011). Performance of cable-stayed bridge pylons subjected to blast loading. Engineering Structures, 33 (4), 1133-1148. 
[5] Griffiths, H., Pugsley, A., Saunders, O. (1968). Report of Injury into the Collapse of Flats at Roman Point, Canning Town. HMSO.

[6] Shi, Y., Li, Z.X., Hao, H. (2009). Numerical investigation of blast-loads on RC slabs from internal explosion. International Workshop on Structures Response to Impact and Blast, 15-17 November 2009, Israel (CD Proceedings).

[7] UFC-3-340-02. (2008). Structures to Resist the Effect of Accidental Explosions. US Department of the Army, Navy and Air Force Technical Manual.

[8] American Society of Civil Engineers (ASCE). (2007). Blast Standard. Ballot version 1, Washington, D.C.

[9] Dragos, J., Wu, C., Oehlers, D.J. (accepted 2012a). Simplification of fully confined blasts for structural response analysis. Engineering Structures.

[10] Smith, P.D., Hetherington, J.G (1994). Blast and Ballistic Loading of Structures. Butterworth-Heinemann, Oxford.

[11] Krauthammer, T., Astarlioglu, S., Blasko, J., Soh, T.B., Ng, P.H. (2007). Pressure-impulse diagrams for the behavior assessment of structural components. International Journal of Impact Engineering, 35 (8), 771-783.

[12] Li, Q.M., Meng, H. (2002a). Pressure-impulse diagram for blast loads based on dimensional analysis and single-degree-of-freedom model, Journal of Engineering Mechanics, 128 (1), 87-92.

[13] Li, Q.M., Meng, H. (2002b). Pulse loading shape effects on pressure-impulse diagram of an elastic-plastic, single-degree-of-freedom structural model, International Journal of Mechanical Sciences, 44 (9), 1985-1998.

[14] Fallah, A.S., Louca, L.A. (2007). Pressure-impulse diagrams for elastic-plastic-hardening and softening single-degree-of-freedom models subjects to blast loading. International Journal of Impact Engineering, 34 (2007), 823-842.

[15] Youngdahl, C.K. (1970). Correlation parameters for eliminating the effect of pulse shape on dynamic plastic deformation. ASME, Journal of Applied Mechanics, 37 (3), 744-752.

[16] Shi, Y.C., Hao, H., Li, Z.X. (2008). Numerical derivation of pressure-impulse diagrams for prediction of RC column damage to blast-loads. International Journal of Impact Engineering, 35 (11), 1213-1227.

[17] Huang, X., Ma, G.W., Li, J.C. (2010), Damage Assessment of Reinforced Concrete Structural Elements Subjected to Blast Loads. International Journal of Protective Structures, 1 (1), 21-43.

[18] Dragos, J., Wu, C., Haskett, M., Oehlers, D. (2013). Derivation of Normalized Pressure Impulse Curves for Flexural Ultra High Performance Concrete Slabs. Journal of Structural Engineering, ASCE, 139 (6), 875-885

[19] Mays, G.C., Smith, P.D. (1995). Blast Effects on Buildings - Design of Buildings to Optimize Resistance to Blast Loading. Thomas Telford, London. 